\title{
EFFECT OF THE GEOMETRICAL PARAMETERS IN A DOMESTIC BURNER WITH CRESCENT FLAME CHANNELS FOR AN OPTIMAL TEMPERATURE DISTRIBUTION AND THERMAL EFFICIENCY
}

\author{
Ramazan Şener ${ }^{1}$, Mehmed R. Özdemir ${ }^{1}$ and Murat U. Yangaz ${ }^{1, *}$
}

\begin{abstract}
Domestic cookers are common tools of house appliances in the world and they have significant share in global energy consumption. Therefore, a small amount of improvement in efficiency would result in a huge drop in total energy and resource activity. This study aims at presenting numerically the thermal efficiency of a domestic burner with crescent-shaped flame channels by changing the distance from the cooker to the burner head and the diameter of the burner. The energy efficiency parameter was evaluated analyzing temperature distribution along the bottom surface of the cooker and unburnt $\mathrm{HC}, \mathrm{CO}$ and $\mathrm{NO}$ emissions. Simulations have been carried out with methane as fuel for three different diameter and distance parameters. The results showed that the temperature on the surface and the emission values of unburnt $\mathrm{CO}, \mathrm{NO}$ and $\mathrm{HC}$ decreased with increasing the cooker diameter and distance parameter.
\end{abstract}

Keywords: Combustion, CFD, Domestic Burner, Cooker, Emissions, Fuel

\section{INTRODUCTION}

The burners are widely used for heating and cooking purposes in domestic and industrial applications due to their simplicity, low cost and ease of programmability [1]. This common utilization causes a great portion of total energy consumption via cookers in the global arena [2,3]. Thereby, a small improvement in combustion characteristics can provide a significant enhancement on the fuel economy and emission [4]. A considerable amount of literature has been established due to the importance of this topic [1-18].

Lucky et al. [3] examined the cooker performance using biomass and natural gas as a fuel. They utilized two types of pan geometry namely round-bottomed pot and flat-bottomed pan. They stated that improving cooker system geometry increased the thermal efficiency of the cooker. The round-bottomed pot exhibited superior thermal performance compared to flat-bottomed pan for all cases. In another study, Ko and Lin [5] investigated the effect of natural gas composition on burner performance in domestic gas burners for various heating power values. They reported that the thermal efficiency and $\mathrm{CO}$ emissions are highly dependent to heating power. The higher heating value operation results in a decrease in thermal efficiency and an increase in $\mathrm{CO}$ emission.

Hou et al. [6] investigated the influence of five significant parameters; swirl flow, loading height, primary aeration, gas flow rate, and gas supply pressure and semi-confined combustion flame on the thermal efficiency and $\mathrm{CO}$ emissions of domestic gas burners. They found that the swirl flow burner (SB) provides higher thermal efficiency but emits slightly higher $\mathrm{CO}$ emission than the conventional radial flow burner (RB). The thermal efficiency of the semi-confined combustion flame SB provides a quite higher thermal efficiency, by about $12 \%$, than open flame RB. The porous medium gas burner systems have been also examined in the literature [1]. The authors designed a compact efficient combined heating system based on the $8 \mathrm{~kW}$ gas porous medium burner coupled with a heat exchanger. The burner is incorporated into space and domestic water heating system for house application. The results indicated that the porous burner provides lower emission values of $\mathrm{CO}$ and NO up to $12.5 \%$. The performance of domestic gas oven has been analyzed numerically by Mistry et al. [7]. They validated their transient numerical model with experiments. The CFD results has agreed to experimental data less than $6 \%$ error.

Muthukumar and Shyamkumar [4] carried out an experimental study to analyze thermal efficiency of a porous radiant burner using LPG as a fuel for different equivalence ratio and power values. The porosity of the burner has been varied from $80 \%$ to $90 \%$ with $5 \%$ increment. They reported that achieving $10 \%$ efficiency 
improvement is possible using porous burner. In another research, Strojnik et al. [16] presented the combustion evolution for gas burner. The visualization study enabled to implement a more effective and safe design of the electric spark sequence, fuel influx ducts, and the combustion chamber configuration. Boggavarapu et al. [2] studied both experimentally and numerically for liquefied petroleum gas (LPG) and piped natural gas (PNG) fuels for a burner. Based on the results of the CFD model, they proposed circular insert and radiant sheet burner types. They reported that these designs have higher thermal efficiency compared to conventional designs. Their experiment results confirmed that the proposed designs increased burner thermal efficiency of $2.5 \%$ for LPG and $10 \%$ for PNG.

Liu et al. [8] performed three-dimensional numerical simulations to optimize the geometrical parameters of a fuel-staged low NO gas burner. The authors validated their numerical data with the experimental measurements of the full-scale burner. The results indicate that the position of the staged gun is the most important factors affecting the $\mathrm{NO}$ emission. Other important factors are stated as gas injection angle and primary gas injection angle by authors.

In a recent study, Kotb and Saad [10] compared co and counter-swirl domestic burner with non-swirl burners on thermal efficiency and CO emissions by using LPG as a fuel. They used pot height and the flame stability as the parameters. Both swirl burners are higher thermal efficiency and lower $\mathrm{CO}$ emission than the nonswirl burner under all operation conditions. They also found that the increase in pot height resulted in lower thermal efficiency for all type burners.

The above brief literature review reveals that while there are a lot of literature studies for gas burners, the studies about the effect of the crescent channel on combustion efficiency, unburnt $\mathrm{HC}, \mathrm{CO}$ and $\mathrm{NO}$ emission, and temperature distribution is inadequate. Furthermore, this study aims at investigating the effect of diameter of burner and height of the cooker on thermal efficiency using methane as a fuel. The effect of different geometries and different parameters on combustion and emission characteristics have been studied.

\section{METHODOLOGY AND DESIGN}

The burner with crescent flame channels is vertically fired and there are 24 channels around the cooker. The crescent flame channels provide more homogenously temperature distribution to the heated surface, see Figure 1. The geometry of the burner was created using a CAD software in Figure 2. Three different cooker bottom wall distances from burner head $(5,10$ and $15 \mathrm{~cm})$ and three different cooker diameters $(25,30$ and $35 \mathrm{~cm})$ were designed in order to analyze the temperature distribution on the surface of the cooker and emission values. Therefore, nine geometries were prepared to examine the effect of distance and diameter of cooker values on the combustion characteristics.

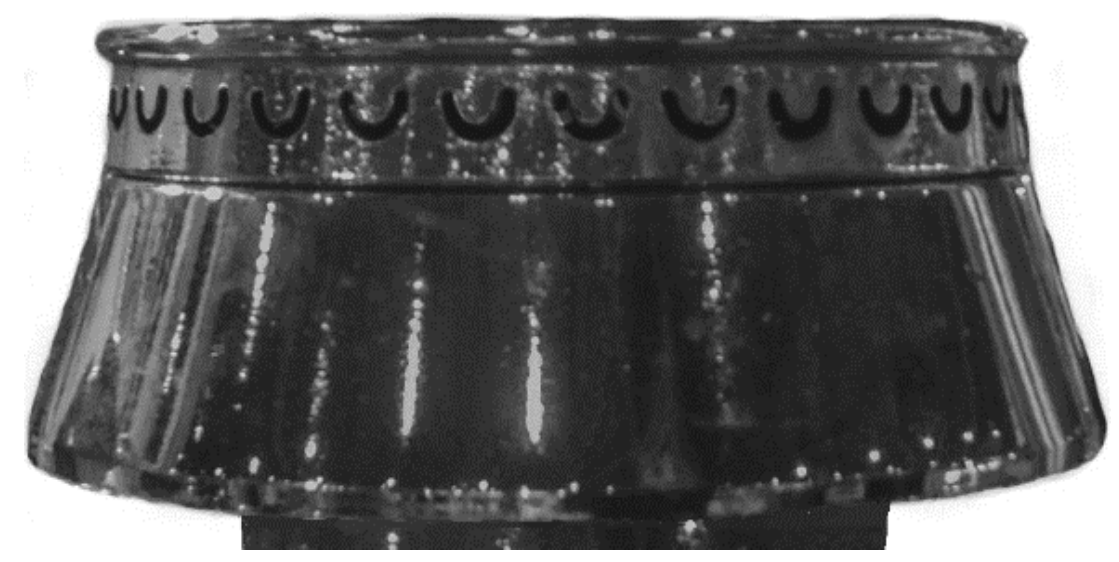

Figure 1. A photograph of the burner with crescent flame channels 


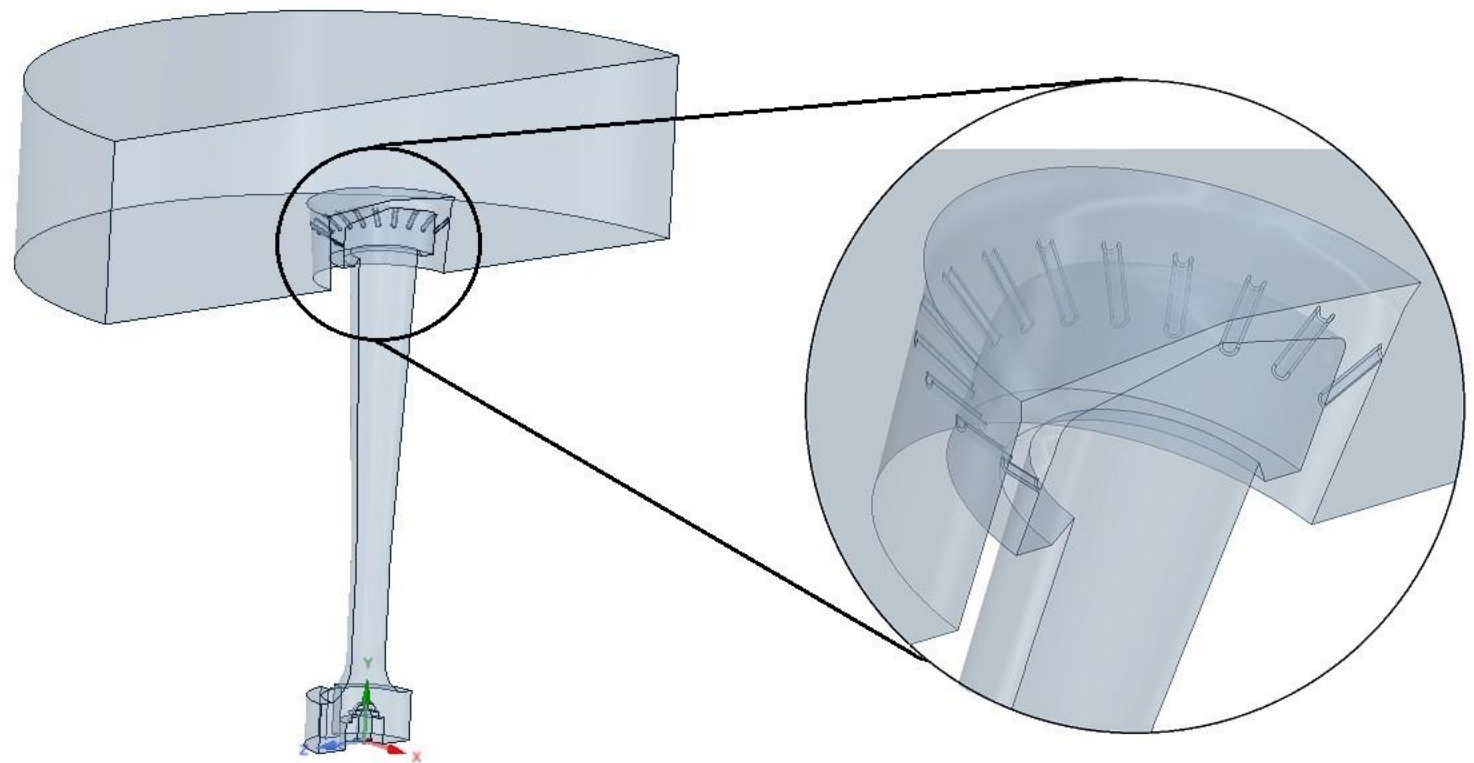

Figure 2. CAD model of the burner with crescent flame channels

\section{NUMERICAL SIMULATION}

Mesh generation is a crucial parameter in numerical studies that might affect the entire performance of the system. Mesh domain of the domestic burner with crescent flame channels was generated using ANSYS Fluent 17.2 software. Because of the complex geometry of the burner, tetrahedral grid type was used. At the top of the domain where the bottom surface of the cooker located, an inflation with five layers applied to the grid. In addition, the grids around the flame channels has been improved by a face sizing with 1.13 growth ratio. The mesh structure of the burner consists of about $4 \mathrm{E}+6$ computational cells (Figure 3 ).

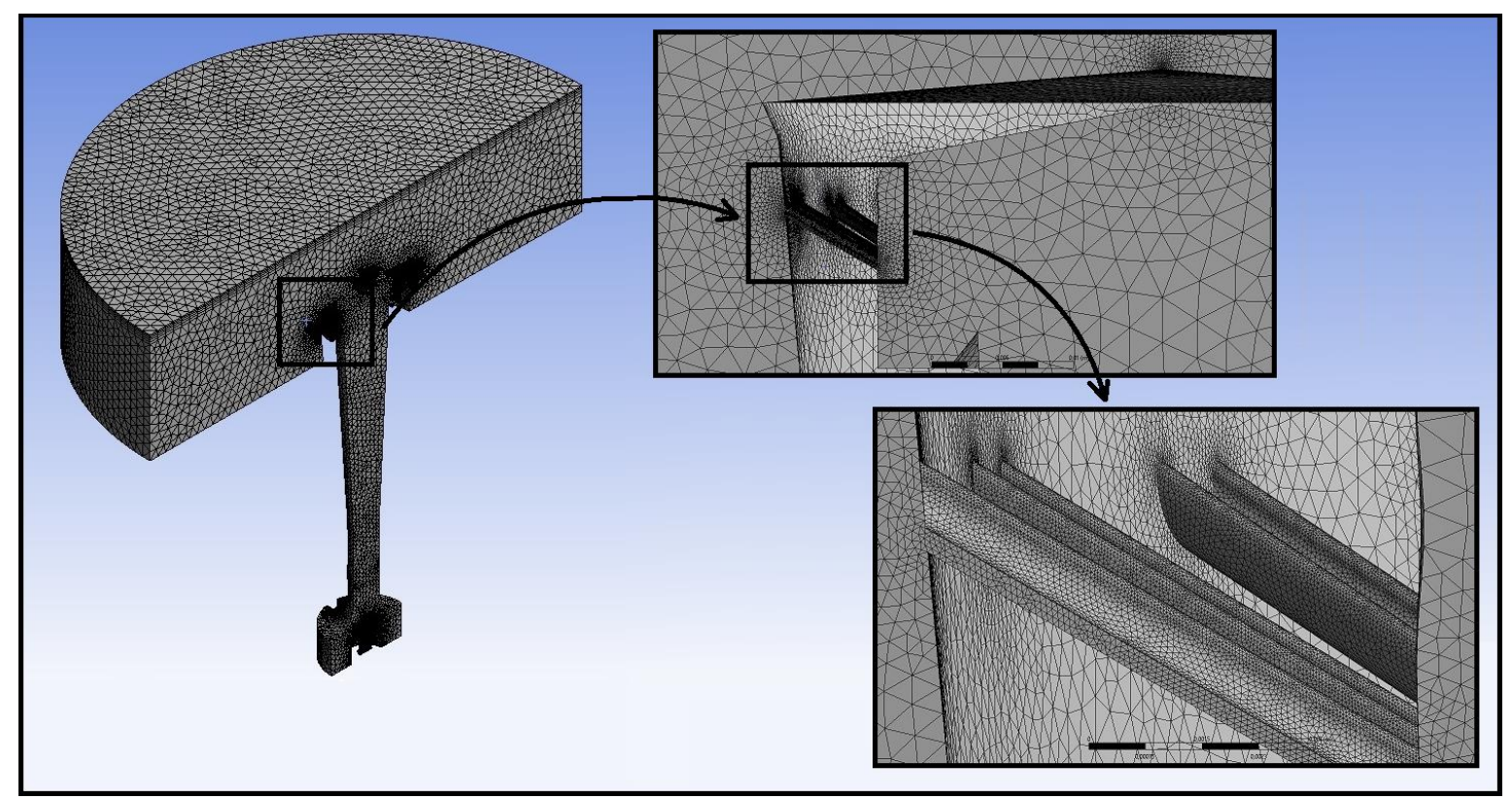

Figure 3. Mesh structure of the domestic burner

Figure 4 shows the results of the mesh independency simulations. It has been given in two separate graphs. Mesh independency simulations have been carried out with five different grid densities for each of the 
nine design points. Results have been compared in average temperature on the bottom surface of the cooker and mass fractions of $\mathrm{CO}_{2}$ emissions from the outlet boundary. According to the results of the mesh independency, Mesh \#4 for each design point has been selected for the simulations.

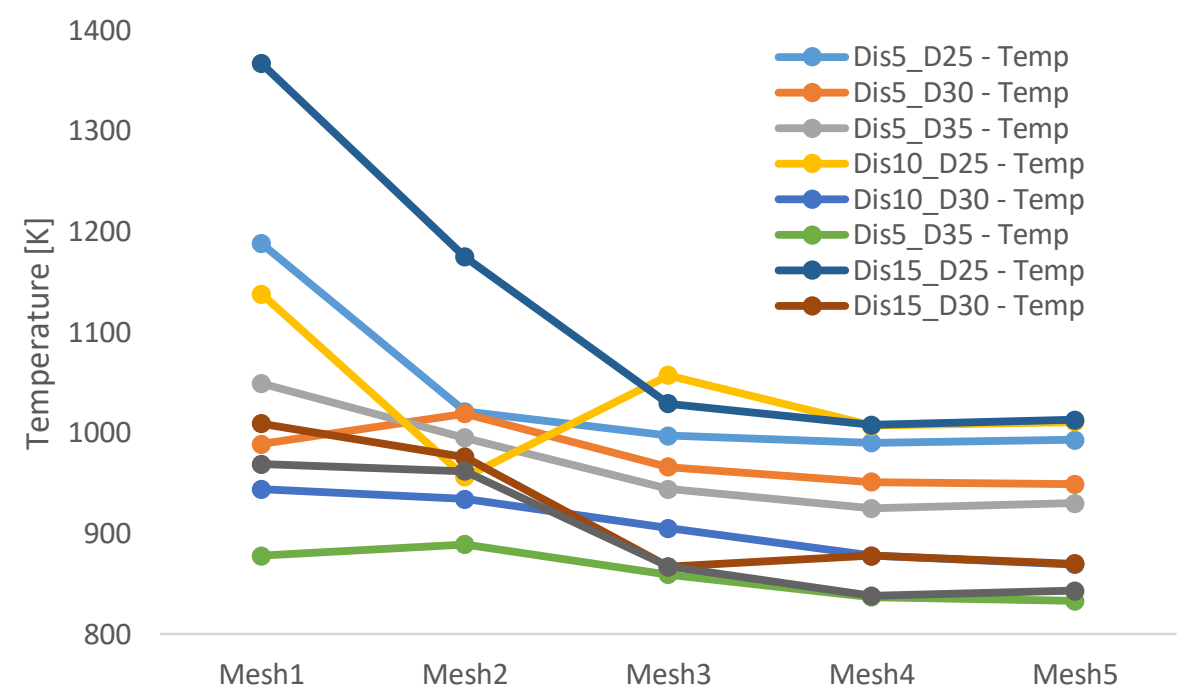

(a)

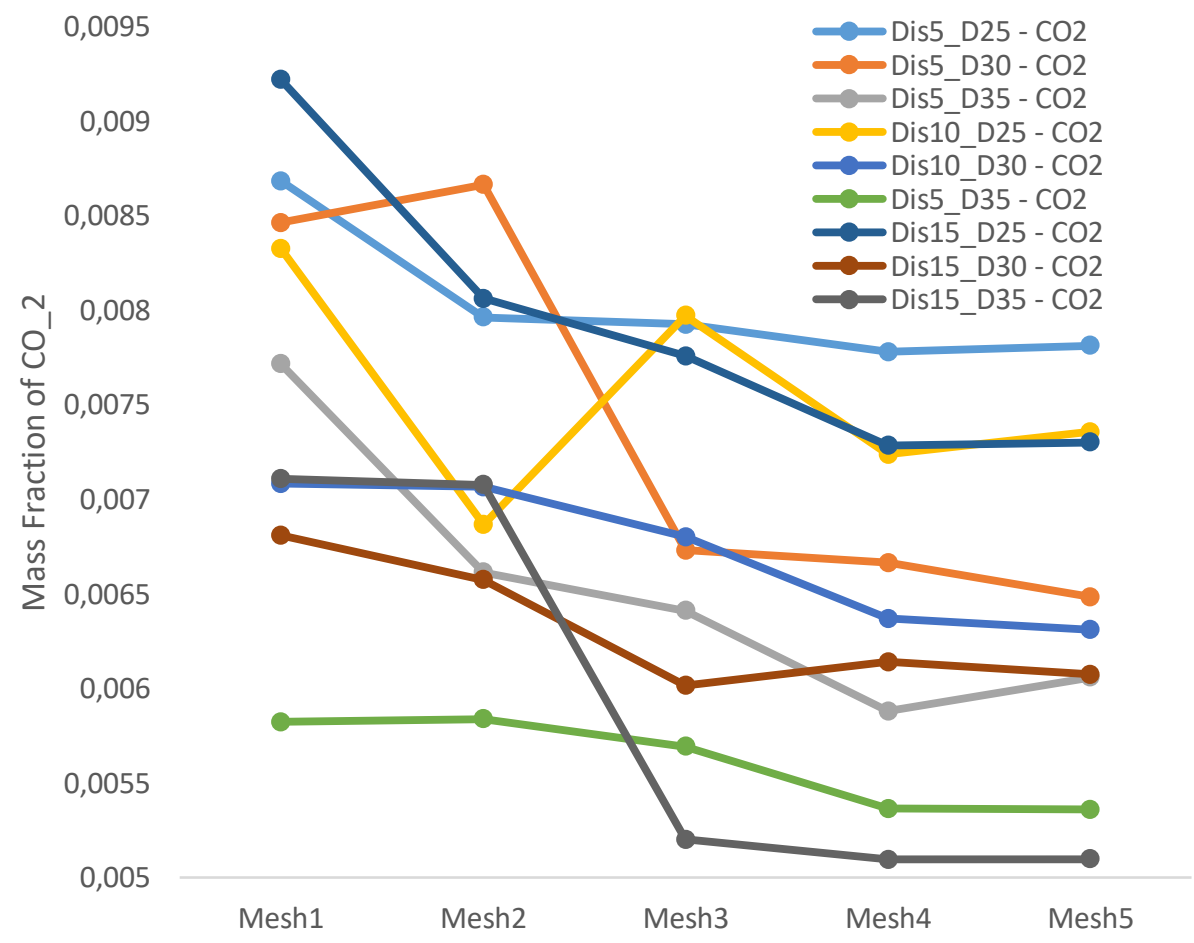

(b)

Figure 4. Results of the mesh independency simulations, a) The variation of temperature for different grid densities, b) The variation in mass fractions of $\mathrm{CO}_{2}$ for different grid densities 


\section{THEORY}

Computational Fluid Dynamics (CFD) simulations were performed using the commercial software ANSYS $^{\circledR}$ Fluent 17.2 that is based on a finite volume method. Governing equations used in the simulations are given below [18]:

The Mass Conservation Equation:

$$
\frac{\partial \rho}{\partial t}+\nabla *(\rho \vec{v})=S_{M}
$$

In Equation $1, \rho, \vec{v}$ symbolize the density and velocity vector respectively. Above equation is the general form of conservation of mass and it is also valid for compressible flows.

The Momentum Conservation Equation:

$$
\frac{\partial}{\partial t}(\rho \vec{v})+\nabla *(\rho \overrightarrow{v v})=-\nabla \mathrm{p}+\nabla *(\overline{\bar{\tau}})+\rho \vec{g}+\vec{F}
$$

In momentum equation, $\frac{\partial}{\partial t}(\rho \vec{v})$ is the unsteady term, which expresses the rate of increase of momentum. $\nabla *(\rho \overrightarrow{v v})$ is the convection term. $\nabla \mathrm{p}$ signify the gradient of pressure. $\rho$ and $\vec{g}$ symbolize dynamic viscosity and gravitational force, respectively. Finally, $\overrightarrow{S_{M}}$ contains the rest of the source terms, which include body forces such as electromagnetic, centrifugal and coriolis forces (Equation 2).

Energy Equation:

$$
\frac{\partial}{\partial t}(\rho e)-\nabla *(\rho e \vec{v})=\frac{\partial p}{\partial t}+\nabla *(k \nabla T)+S_{e}+S_{\text {rad }}
$$

The energy equation can be derived from the first law of thermodynamics. Its physical meaning is that rate of increase in internal energy equals to the sum of net rate of heat added and net rate of work done. $\frac{\partial}{\partial t}(\rho e)$ stands for local energy change with time. $\nabla *(\rho e \vec{v})$ term is convection term and $e$ represents the internal energy. $\nabla *(k \nabla T), S_{r a d}$ and $S_{e}$ terms are heat flux (diffusion) and source term in energy equation (Equation 3 ).

Realizable k-ع turbulence model, and transport equation for the model is given below [19]:

$k e q n:$

$$
\frac{\partial}{\partial t}(\rho k)+\frac{\partial}{\partial x_{i}}\left(\rho k u_{i}\right)=\frac{\partial}{\partial x_{j}}\left(\left(\mu+\frac{\mu_{t}}{\sigma_{\varepsilon}}\right) \frac{\partial k}{\partial x_{j}}\right)+P_{k}+P_{b}-\rho \varepsilon-Y_{M}+S_{k}
$$

and

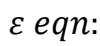

$\frac{\partial}{\partial t}(\rho \varepsilon)+\frac{\partial}{\partial x_{i}}\left(\rho \varepsilon u_{i}\right)=\frac{\partial}{\partial x_{j}}\left(\left(\mu+\frac{\mu_{t}}{\sigma_{\varepsilon}}\right) \frac{\partial \varepsilon}{\partial x_{j}}\right)+\rho C_{1} S_{\varepsilon}-\rho C_{2} \frac{\varepsilon^{2}}{k+\sqrt{\vartheta \varepsilon}}+C_{1 \varepsilon} \frac{\varepsilon}{k} C_{3 \varepsilon} P_{b}+S_{\varepsilon}$

where

$$
C_{1}=\max \left[0.43, \frac{\eta}{\eta+5}\right]
$$

and 


$$
\eta=\mathrm{S} \frac{k}{\varepsilon}, S=\sqrt{2 S_{i j} S_{i j}}
$$

in Realizable k- $\mathrm{\varepsilon}$ turbulence model, turbulent (eddy) viscosity can be calculated using the below formula:

$$
\mu_{t}=\rho C_{\mu} \frac{k^{2}}{\varepsilon}
$$

In Equation 4, the first term is the rate of change of turbulent kinetic energy, second term on the left hand side is the transport of turbulent kinetic energy $(\mathrm{k})$ by convection. On the other hand, the first term on the right hand side is the transport of $\mathrm{k}$ by both viscous and Reynolds stress or the diffusion term, $P_{k}$ symbolizes the production of turbulent kinetic energy. $P_{b}$ is the production of turbulent kinetic energy due to buoyancy, fourth term on the right hand side represents the destruction of $\mathrm{k}, Y_{M}$ is the fluctuating dilatation contribution in compressible turbulence to the overall dissipation rate. And $S_{k}$ is a source term. Equation 5 can be explained as the rate of change of $\varepsilon$ summation with transport of $\varepsilon$ by convection equals to the transport of $\varepsilon$ by diffusion and production rate of $\varepsilon$ difference by destruction rate of $\varepsilon$.

Due to high computational costs, realizable $\mathrm{k}-\varepsilon$ model has been opted instead of using a non-linear eddy viscosity model. Because of the realizable characteristics of the model, it satisfies some mathematical constraints on Reynolds stresses and therefore it is consistent with turbulent flow physics. It also predicts the spreading rate of round jets more accurately. It has been tested extensively and validated for boundary layer and channel flows, mixing layers and jets and free flows [20].

The mass fractions of species in a reacting flow of gases can be calculated by solving the species continuity equation given below [21]:

Species Continuity Equation:

$$
\frac{\partial}{\partial t}\left(\rho_{i}\right)+\nabla *\left[\rho_{i}\left(\vec{v}+\vec{u}_{i}\right)\right]=\dot{\omega}_{i} \quad i=1,2,3, \ldots \ldots \ldots n
$$

In the above equation, $\rho_{i}$ is the density of the species. $\vec{v}$ is the velocity vector. $\vec{u}_{i}$ symbolizes the diffusion velocity of the species and the last term, $\dot{\omega}_{i}$ is the production rate of the species due to the chemical reactions.

Combustion can be defined as the exothermic reaction between a fuel and an oxidizer. There are three elements to start the combustion reactions: the fuel, oxidizer and an ignition source. Generally, hydrocarbons $\left(\mathrm{C}_{\mathrm{x}} \mathrm{H}_{\mathrm{y}}\right)$ are the common fuels used in both large and small scale combustions systems. The ignition source can be an external source such as in spark-ignition systems or it may be provided via a mechanism, which is based on an auto ignition concept (i.e. compression ignition engines).

Ideal combustion equation of methane with air as the oxidizer is given below:

$$
\mathrm{CH}_{4}+2\left(\mathrm{O}_{2}+3.76 \mathrm{~N}_{2}\right) \rightarrow \mathrm{CO}_{2}+2 \mathrm{H}_{2} \mathrm{O}+7.52 \mathrm{~N}_{2}
$$

Combustion products may include carbon monoxide $(\mathrm{CO})$, carbon dioxide $\left(\mathrm{CO}_{2}\right), \mathrm{H}_{2} \mathrm{O}, \mathrm{NO}_{x}$ and other compounds.

Combustion is divided into three categories depending on how the oxidizer and the fuel is mixed:

- Non-premixed - fuel and the oxidizer are fed into the combustion chamber in separate channels and the reaction starts where they meet first.

- Partially-premixed - a portion of the oxidizer is mixed with the fuel before entering the combustion chamber and the rest of the oxidizer is supplied into the chamber via separate channel.

- Premixed - the mixture of fuel and oxidizer is formed before entering the combustion chamber. 
Non-premixed model has been used for modelling combustion in the system. It is based on mixture fraction theory. The solution technique utilizes the probability density function (PDF), which is a statistical method. The reaction chain consists 18 species in the simulations including the compound NO.

An implicit solver was selected. The solutions have been carried out by using a pressure-based and steady-state setup. In the nonlinear governing equations, the convection terms were discretized by a second order upwind scheme and the diffusion terms by a second order central-difference scheme. The relationship between velocity and pressure corrections was solved by the Coupled algorithm. The appropriate under-relaxation factors were applied to avoid instability in the solution.

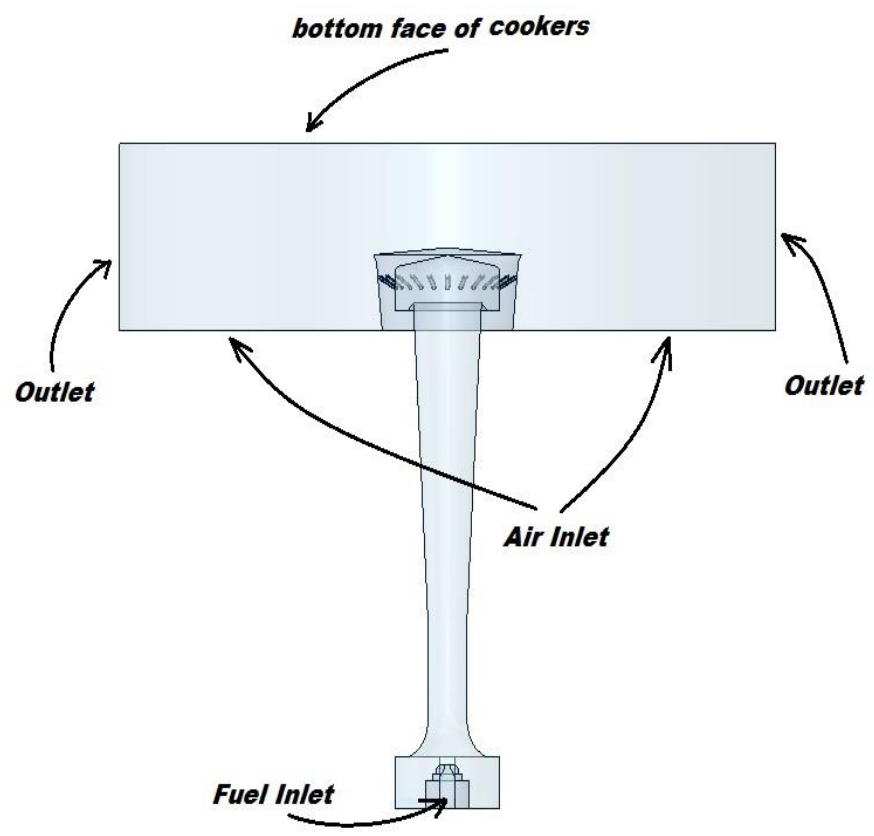

Figure 5. Locations of the boundary conditions on the solution domain

Two convergence criteria were used in this study. The first is to achieve an asymptotic behavior of the residuals of the governing equations. The convergence criteria were set to $1 \mathrm{E}-5$ for the continuity, momentum, TKE, dissipation rate of the TKE, and mixture fraction. The convergence criteria were set to 1E-6 for the energy and RTE. The boundary conditions are illustrated schematically in Figure 5.

Table 1. Details of the parametric study

\begin{tabular}{|c|c|c|}
\hline Designs & Distance (cm) & Diameter (cm) \\
\hline Dis5_D25 & 5 & 25 \\
\hline Dis5_D30 & 5 & 30 \\
\hline Dis5_D35 & 5 & 35 \\
\hline Dis10_D25 & 10 & 30 \\
\hline Dis10_D30 & 10 & 35 \\
\hline Dis10_D35 & 10 & 25 \\
\hline Dis15_D25 & 15 & 30 \\
\hline Dis15_D30 & 15 & 35 \\
\hline Dis15_D35 & 15 & \\
\hline
\end{tabular}


Table 2. Operation and boundary conditions of the solutions

\begin{tabular}{|c|c|}
\hline Temperature (outlet) & $298 \mathrm{~K}$ \\
\hline Atmospheric Pressure & $101325 \mathrm{~Pa}$ \\
\hline Fuel Inlet Pressure & $2100 \mathrm{~Pa}$ (gauge pressure) \\
\hline Fuel Inlet Temperature & $300 \mathrm{~K}$ \\
\hline Fuel Inlet hydraulic diameter & $4,278 \mathrm{~mm}$ \\
\hline Air inlet pressure & $101325 \mathrm{~Pa}$ \\
\hline Air inlet Temperature & $300 \mathrm{~K}$ \\
\hline Air inlet hydraulic diameter & $140 \mathrm{~mm}$ \\
\hline Outlet hydraulic diameter & 25,30 and $35 \mathrm{~cm}$ \\
\hline Cooker Bottom Wall material & Steel \\
\hline Excess Air Ratio & $10 \%$ \\
\hline
\end{tabular}

Furthermore, it provides to examine the mass and energy balance. Iterations were continued until differences were less than $1 \mathrm{E}-6 \mathrm{~kg} / \mathrm{s}$. The two criteria are sufficient for a good convergence [8]. Methane, which is quite common for domestic applications, was used as a fuel. The fuel inlet pressure was selected as $2100 \mathrm{~Pa}$ due to the applicability as a home-line pressure. Other boundary and operation conditions and solution settings are presented in Table 1 and Table 2. Parametric solutions were simulated according to different distances between cooker bottom wall and burner head $(5,10$ and $15 \mathrm{~cm})$ and cooker diameters $(25,30$ and $35 \mathrm{~cm})$. All the design parameters and their abbreviations have been given in Table 1. The cooker located on top of the burner and controlled by the temperature. It must not exceed safe operating temperature, according to a material of the cooker. In our design, different fuels such as natural gas, biogas, hydrogen, LPG could be used. Natural gas used and it mostly consist of methane. Therefore, a hundred percent of methane was used for simulations.

\section{RESULTS AND DISCUSSION}

In this section, the results and inferences of numerical studies are presented. CFD simulations describe the thermal efficiency, temperature distribution and emission features of the domestic burner.

Figure 6 demonstrates temperature distribution on the bottom surface of the cooker. For all cookers, it can be observed that the edge of the cookers is hotter than the middle part of cookers. It can also be clearly seen that the designs Dis10_D25 and Dis15_D25 yielded higher temperature compared to other designs. The maximum temperature values are $1007 \mathrm{~K}$ and $1008 \mathrm{~K}$ respectively. On the other hand, lower temperature values are obtained for the Dis10_D35 and Dis15_D35 designs. The maximum temperature values for the designs are found to be $837 \mathrm{~K}$ and $838 \mathrm{~K}$, respectively.

It can be inferred that there is an inverse relationship between diameter and temperature. In another word, the temperature is decreasing with the increase in diameter. These findings are not surprising. Since the surface area increases with the increase in diameter, the cookers having higher surface area cool faster in a specified time. Moreover, when the diameter increases, temperature distribution along the bottom surface of the cooker becomes more homogenously as it is expected due to the increased heat transfer area.

The temperature distribution of cross-sectional area of the whole burner body is presented in Figure 6. It can be clearly seen that the hot spots on the contour reach to the bottom surface of the cooker. The similar results are obtained in all solutions where the hot spots starting to appear closer to the cooker bottom surface. Temperature contours give a general view of the forms of the flames. 


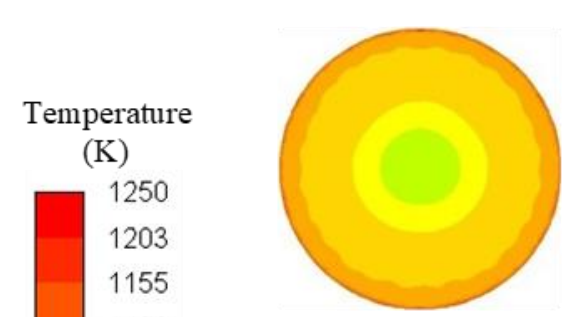

1108 Dis5_D25

1060

1013

965

917.5

870

822.5

775

727.5

680

632.5

585

537.5

490

442.5

395

347.5

300

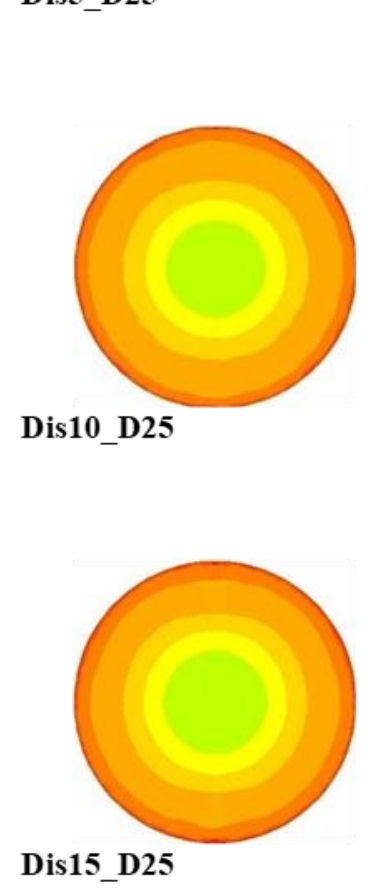

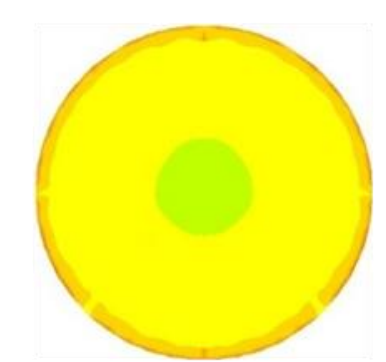

Dis5_D30

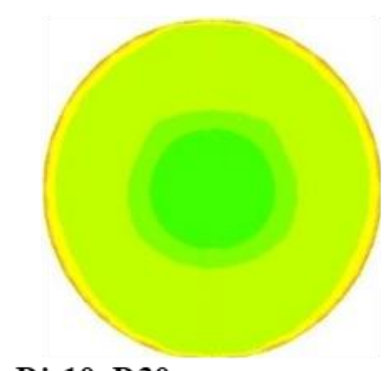

Dis10_D30

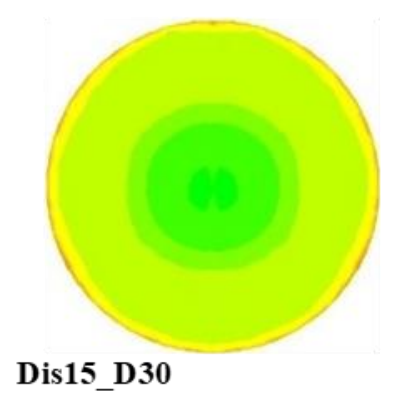

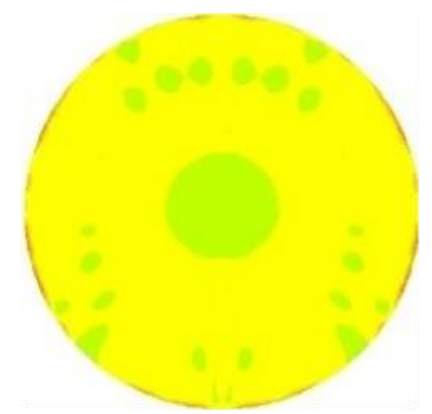

Dis5_D35

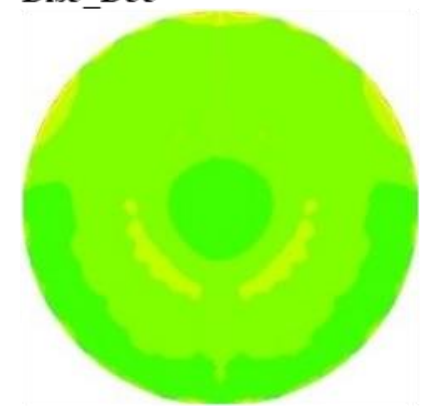

Dis10 D35

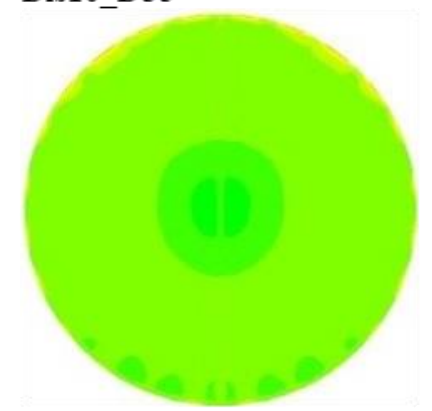

Dis15_D35

Figure 6. Temperature distribution on the cooker's bottom surfaces

Figure 7 shows the average temperatures at the bottom wall of the cooker and the outlet boundary. It can be seen that when the diameter increases, temperature values on the bottom surface of the cooker decreases which is mainly due to the enlarged outlet surface area where the higher convective heat transfer occurs to the ambient air. Another factor could be the temperature difference that is the driving force in heat transfer. The ambient air is kept constant as $300 \mathrm{~K}$ during simulations. Also, the minimum average temperature is found to be around $830 \mathrm{~K}$ at the bottom wall. Therefore, the heat transfer rate will be faster from the flame core to the outlet than to the cooker.

In the literature, the emission values from the domestic burners were found to be quite low [17]. Furthermore, the simulation results reveal that the emission values are inversely proportional with the cooker diameter. The emission values decreased considerably when the cooker diameter was increased. Also, it was found that the distance from the cooker to the burner head has an influence on emission values. As the distance becomes larger the emission values of $\mathrm{HC}, \mathrm{CO}$, and $\mathrm{CO}_{2}$ reduced.

Figure 8 demonstrates the emission values from the outlet boundary to the ambient air in terms of mass fractions. As it can be seen from the graph both the distance and the diameter decrease the emissions. Moreover, it can be observed that the effects of the distance and the diameter are much pronounced on CO levels than on $\mathrm{HC}$ emissions. The general trend is that when the temperature on the bottom surface increase, $\mathrm{CO}_{2}$ emission levels also increase. This behavior might be attributed to the fact that the activation energy phenomenon of the mixture. The mixture could reach the activation energy faster in order to initiate the chemical reactions. 


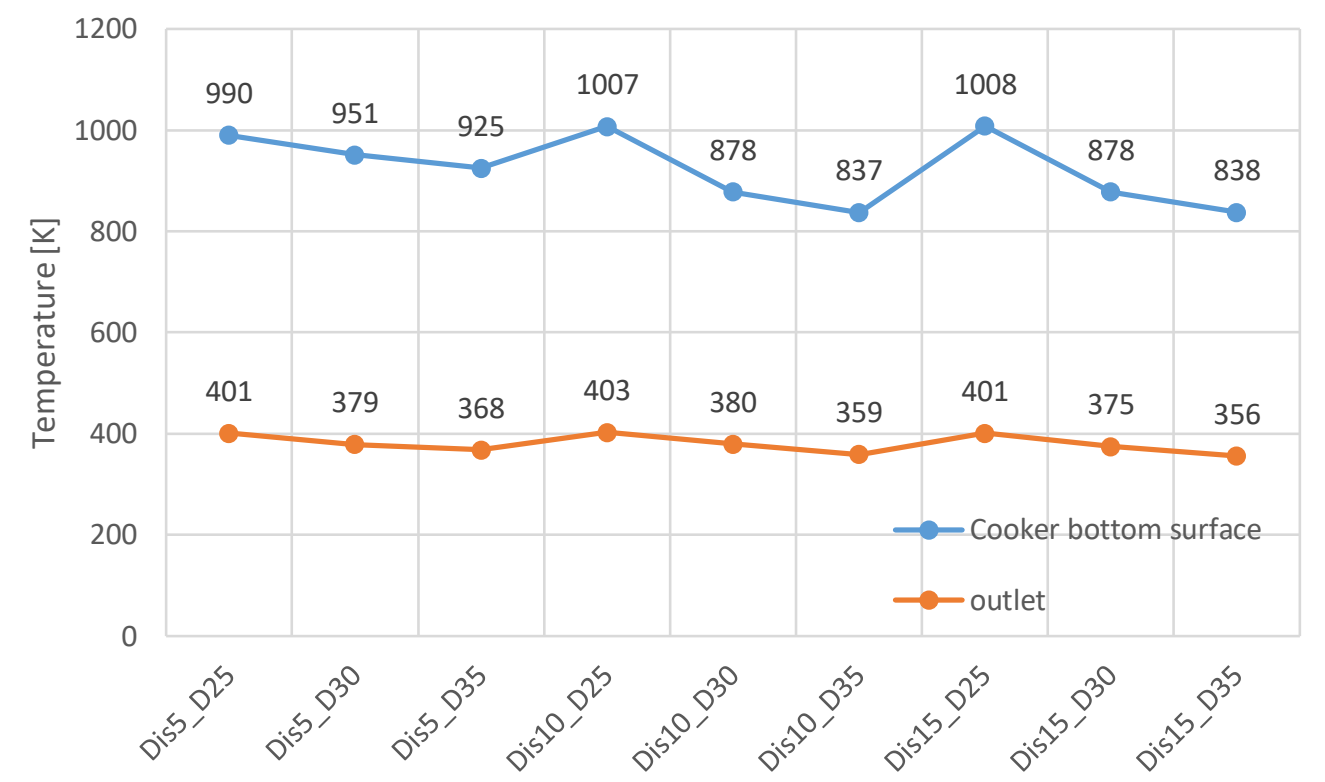

Figure 7. Average temperature values both at the bottom surface of the cooker and the outlet boundary

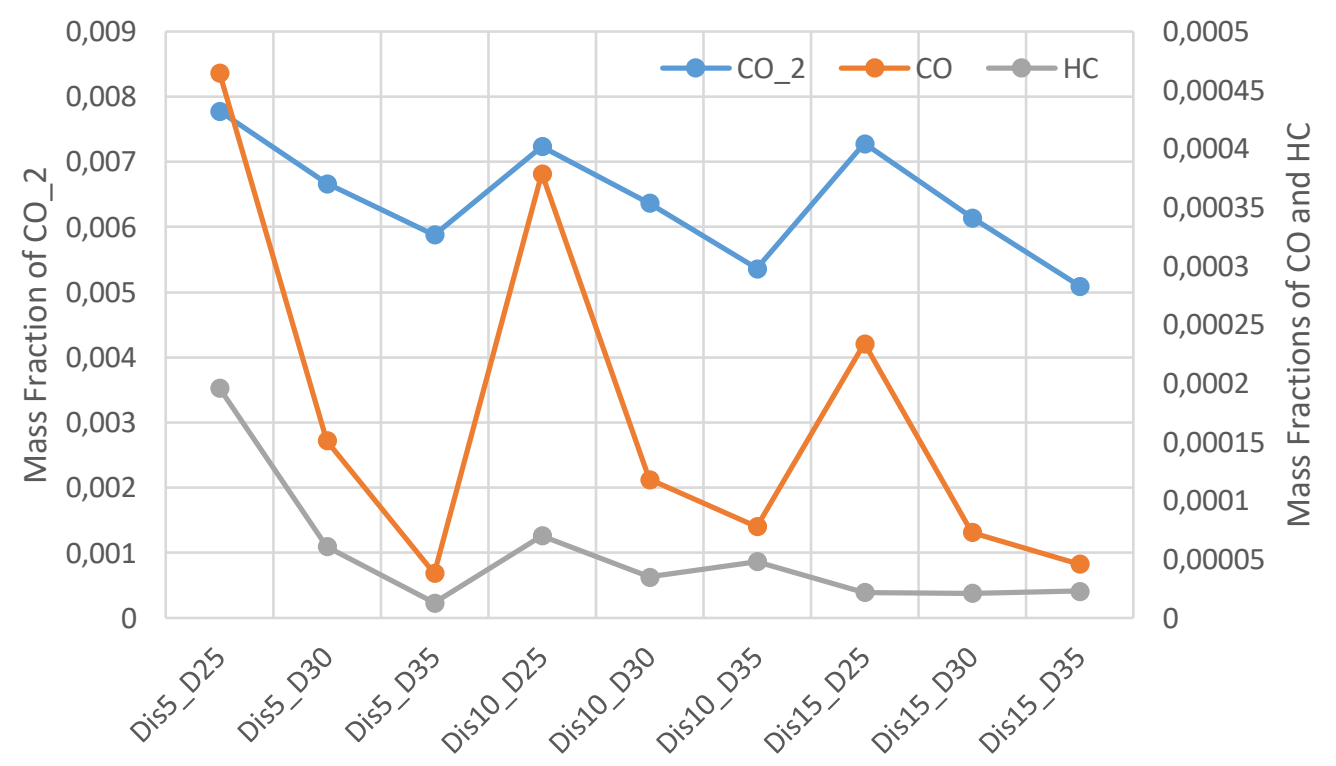

Figure 8. $\mathrm{CO}_{2}, \mathrm{CO}$ and $\mathrm{HC}$ emission values from the outlet boundary

In addition to this, it can be also seen from the Figure 8 that the flame core temperatures reach almost $2100 \mathrm{~K}$ values. Figure 8 also presents the distance flame temperature relationship. As the distance gets higher, the flame temperatures get higher as well. This situation can be explained by the increased volume between the flame channels and the bottom wall of the cooker, which provides more space for better mixing. Thereby, the mixture does not leave from the unreacted computational domain. 


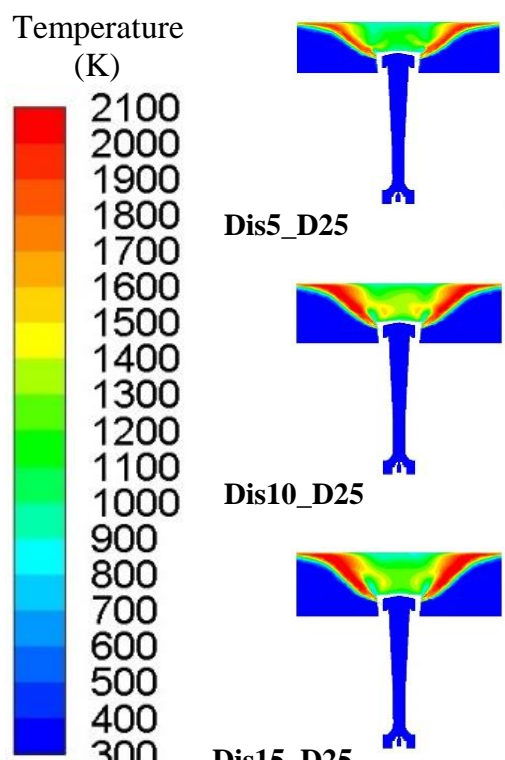

Dis15_D25

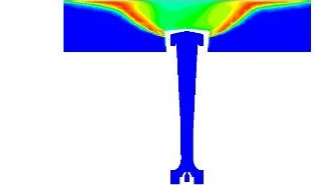

Dis5_D30

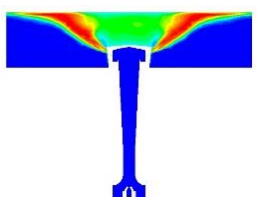

Dis10_D30

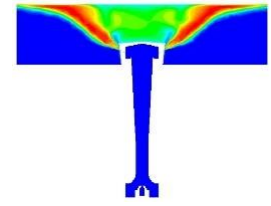

Dis15_D30

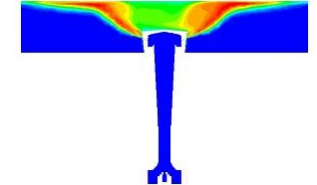

Dis5_D35

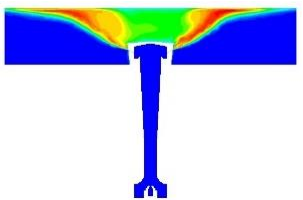

Dis10_D35

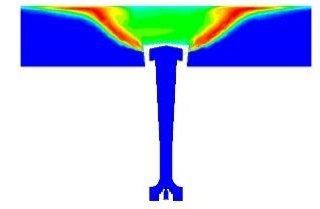

Dis15 D35

Figure 9. Temperature distribution on the whole burner body

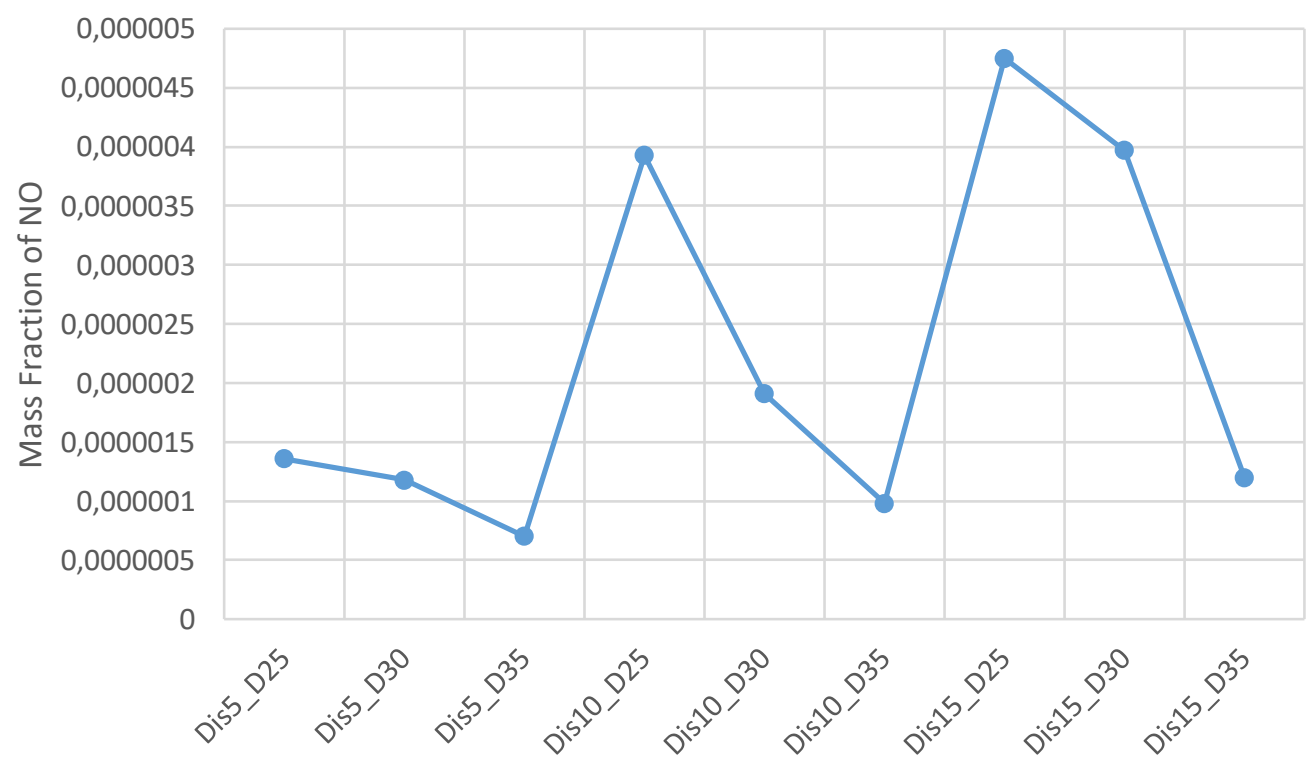

Figure 10. Emission values from the outlet boundary

The NO emission values are plotted in the Figure 10. Figure 10 reveals that the NO levels appear to be compliant with the temperature values, which means that the effect of thermal NO mechanism (Zeldovich mechanism) [22,23] are dominant in the simulations. If the temperature exceeds the $1200 \mathrm{~K}$ value the dominant reaction pathway becomes the thermally dominated NO formation.

\section{CONCLUSION}

In this study, it has been aimed to investigate the effect of cooker diameter and distance from the cooker to the burner head on the temperature and emission values parametrically. As known, development of experimental case and examination of the effect of different parameters is a difficult, costly and time-consuming procedure. For this reason, CFD methods can be used to reduce additional costs without influencing the accuracy 
of the results. Simulations have been carried out using methane, which is one of the most commonly used fuel in the domestic burners.

According to the results, the increase in diameter causes a significant decrease in the temperature owing to the increased surface area of the outlet boundary. In addition, temperature difference may have the leading role in here. Because it directly affects the heat transfer rates. The increase in diameter and distance also cause a decrease in emission values. This phenomenon can be explained due to the homogenous distribution of temperature and the enhanced volume under the cooker for better mixing. Since NO levels are rather low in this domestic type of burners, our main concern was on the emissions of $\mathrm{CO}$ and unburnt $\mathrm{HC}$, which directly represent the combustion efficiency.

In future studies, convection from heated substances inside the cooker can be included in simulation to get more realistic solution. The optimization study can be performed in burner channels to achieve more homogeneous combustion and more reduced $\mathrm{CO}, \mathrm{HC}$ and $\mathrm{NO}$ emission values. In addition, more homogenous flame distribution on the cooker surface can be obtained using porous burner geometry. Moreover, swirl effect could be utilized for enhancing the combustion efficiency and lowering the emissions further.

\section{NOMENCLATURE}

$\mathrm{a}_{1}$
$\mathrm{C}_{1}, \mathrm{C}_{2}$
$\mathrm{C}_{1 \varepsilon}, \mathrm{C}_{3 \varepsilon}$
$\mathrm{C}_{\mu}$
$\mathrm{e}$
$\mathrm{F}$
$\mathrm{F}_{1,2}$
$\mathrm{~g}$
$\mathrm{k}$
$\mathrm{P}$
$\mathrm{P}_{\mathrm{k}}, \mathrm{P}_{\mathrm{b}}$
$\mathrm{S}, \mathrm{S}_{\mathrm{e}}, \mathrm{S}_{\varepsilon}, \mathrm{S}_{\mathrm{M}}, \mathrm{S}_{\mathrm{rad}}$
$\mathrm{S}_{\mathrm{ij}}$
$\mathrm{T}$
$\mathrm{t}$
$\mathrm{U}_{\mathrm{j}}$
$\vec{u}_{i}$
$\mathrm{v}$
$\vec{v}$
$\mathrm{x}$
$\mathrm{Y}_{\mathrm{M}}$

0.31

Constant

Constant

Constant

Internal energy

Force, N

Blending functions

Gravitational acceleration, $\mathrm{m} / \mathrm{s}^{2}$

Turbulent kinetic energy, $\mathrm{m}^{2} / \mathrm{s}^{2}$

Pressure, $\mathrm{Pa}$

Production term

Source term

Strain tensor

Temperature, $\mathrm{K}$

Time, sec

Axial velocity, $\mathrm{m} / \mathrm{s}$

Diffusion velocity of the species, $\mathrm{m} / \mathrm{s}$

Velocity, $\mathrm{m} / \mathrm{s}$

Velocity vector, $\mathrm{m} / \mathrm{s}$

Position, $\mathrm{m}$

Contribution of the fluctuating of dilatation

Greek symbols

$\alpha$

$\beta^{*}$

Thermal diffusivity

0.09

Rate of dissipation per unit mass, $\mathrm{m}^{2} / \mathrm{s}^{3}$

Collective term

Density, $\mathrm{kg} / \mathrm{m}^{3}$

Surface tension coefficient, $\mathrm{N} / \mathrm{m}$

0.5

0.5

0.856

Stress tensor

Kinematic viscosity, $\mathrm{m}^{2} / \mathrm{s}$

Kinematic turbulent viscosity, $\mathrm{m}^{2} / \mathrm{s}$

Specific dissipation rate, $1 / \mathrm{s}$

Production rate of the species, $\mathrm{mol} / \mathrm{m}^{3} \mathrm{~s}$

Subscripts

$i$

Refers to the specie 


\section{REFERENCES}

[1] F. Avdic, M. Adzic, F. Durst, Small scale porous medium combustion system for heat production in households, Appl. Energy. 87 (2010) 2148-2155. doi:10.1016/J.APENERGY.2009.11.010.

[2] P. Boggavarapu, B. Ray, R. V. Ravikrishna, Thermal efficiency of LPG and PNG-fired burners: Experimental and numerical studies, Fuel. 116 (2014) 709-715. doi:10.1016/j.fuel.2013.08.054.

[3] R. Akter Lucky, I. Hossain, Efficiency study of Bangladeshi cookstoves with an emphasis on gas cookstoves, Energy. 26 (2001) 221-237. doi:10.1016/S0360-5442(00)00066-9.

[4] P. Muthukumar, P.I. Shyamkumar, Development of novel porous radiant burners for LPG cooking applications, Fuel. 112 (2013) 562-566. doi:10.1016/j.fuel.2011.09.006.

[5] Y.-C. Ko, T.-H. Lin, Emissions and efficiency of a domestic gas stove burning natural gases with various compositions, Energy Convers. Manag. 44 (2003) 3001-3014. doi:10.1016/S0196-8904(03)00074-8.

[6] S.-S. Hou, C.-Y. Lee, T.-H. Lin, Efficiency and emissions of a new domestic gas burner with a swirling flame, Energy Convers. Manag. 48 (2007) 1401-1410. doi:10.1016/J.ENCONMAN.2006.12.001.

[7] H. Mistry, S. Ganapathisubbu, S. Dey, P. Bishnoi, J.L. Castillo, A methodology to model flow-thermals inside a domestic gas oven, Appl. Therm. Eng. 31 (2011) 103-111. doi:10.1016/J.APPLTHERMALENG.2010.08.022.

[8] B. Liu, B. Bao, Y. Wang, H. Xu, Numerical simulation of flow, combustion and NO emission of a fuelstaged industrial gas burner, J. Energy Inst. 90 (2017) 441-451. doi:10.1016/j.joei.2016.03.005.

[9] S. Panigrahy, N.K. Mishra, S.C. Mishra, P. Muthukumar, Numerical and experimental analyses of LPG (liquefied petroleum gas) combustion in a domestic cooking stove with a porous radiant burner, Energy. 95 (2016) 404-414. doi:10.1016/J.ENERGY.2015.12.015.

[10] A. Kotb, H. Saad, Case study for co and counter swirling domestic burners, Case Stud. Therm. Eng. 11 (2018) 98-104. doi:10.1016/J.CSITE.2018.01.004.

[11] İ.B. Özdemir, Simulation of turbulent combustion in a self-aerated domestic gas oven, Appl. Therm. Eng. 113 (2017) 160-169. doi:10.1016/J.APPLTHERMALENG.2016.10.205.

[12] V. Yousefi-Asli, E. Houshfar, F. Beygi-Khosroshahi, M. Ashjaee, Experimental investigation on temperature field and heat transfer distribution of a slot burner methane/air flame impinging on a curved surface, Appl. Therm. Eng. 129 (2018) 761-771. doi:10.1016/J.APPLTHERMALENG.2017.10.084.

[13] S. Panigrahy, S.C. Mishra, The combustion characteristics and performance evaluation of DME (dimethyl ether) as an alternative fuel in a two-section porous burner for domestic cooking application, Energy. 150 (2018) 176-189. doi:10.1016/J.ENERGY.2018.02.121.

[14] N.K. Mishra, P. Muthukumar, Development and testing of energy efficient and environment friendly porous radiant burner operating on liquefied petroleum gas, Appl. Therm. Eng. 129 (2018) 482-489. doi:10.1016/J.APPLTHERMALENG.2017.10.068.

[15] M. Ilbas, S. Karyeyen, An experimental and numerical study on turbulent combustion of hydrogen-rich coal gases in a generated non-premixed burner, Fuel. 194 (2017) 274-290. doi:10.1016/J.FUEL.2017.01.016.

[16] M. Strojnik, G. Paez, M. Scholl, Combustion initiation and evolution during the first $400 \mathrm{~ms}$ in a gas burner at $10 \mathrm{~lm}$, Infrared Phys. Technol. (2013) 42-49.

[17] M.U. Yangaz, CFD Modeling of Gas Burners using Renewable and Fossil Fuels, Marmara University, 2014.

[18] S. Kakaç, A. Pramuanjaroenkij, X.Y. Zhou, A review of numerical modeling of solid oxide fuel cells, Int. J. Hydrog. Energy. (2007). doi:DOI 10.1016/j.ijhydene.2006.11.028.

[19] H.K. (Henk K. Versteeg, W. (Weeratunge) Malalasekera, An introduction to computational fluid dynamics : the finite volume method, Pearson Education Ltd, 2007.

[20] T.-H. Shih, W.W. Liou, A. Shabbir, Z. Yang, J. Zhu, A new k-€ eddy viscosity model for high reynolds number turbulent flows, Comput. Fluids. 24 (1995) 227-238. doi:10.1016/0045-7930(94)00032-T.

[21] D.A. (Dale A. Anderson, R.H. Pletcher, J.C. Tannehill, Computational fluid mechanics and heat transfer, n.d. $\quad$ https://www.crcpress.com/Computational-Fluid-Mechanics-and-Heat-Transfer-ThirdEdition/Anderson-Tannehill-Pletcher/p/book/9781591690375 (accessed May 10, 2018).

[22] T. Rutar, J.C.Y. Lee, P. Dagaut, P.C. Malte, A.A. Byrne, NO $x$ formation pathways in lean-premixedprevapourized combustion of fuels with carbon-to-hydrogen ratio between 0.25 and 0.88 , Proc. Inst. Mech. Eng. Part A J. Power Energy. 221 (2007) 387-398. doi:10.1243/09576509JPE288.

[23] F. BACHMAIER, K.H. EBERIUS, T. JUST, The Formation of Nitric Oxide and the Detection of HCN in Premixed Hydrocarbon-Air Flames at 1 Atmosphere, Combust. Sci. Technol. 7 (1973) 77-84. doi:10.1080/00102207308952345. 